\title{
On Oscillating Carrier Dynamics in Highly Excited InP:Fe Crystals
}

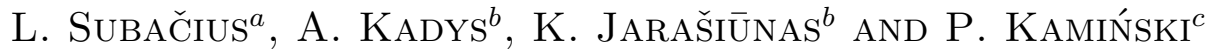 \\ ${ }^{a}$ Semiconductor Physics Institute
}

A. Goštauto 11, LT-01108 Vilnius, Lithuania

${ }^{b}$ Institute of Materials Science and Applied Research, Vilnius University

Saulètekio al. 9-3, LT-10222, Vilnius, Lithuania

${ }^{c}$ Institute of Electronic Materials Technology

Wólczyńska 133, 01-919 Warszawa, Poland

The numerical analysis and experimental data on time-resolved fourwave mixing confirmed a novel origin of oscillations in subnanosecond carrier dynamics in highly excited InP:Fe crystals. The effect was attributed to simultaneous presence of electron and hole gratings, which drift in the space charge field and contribute constructively or destructively to refractive index modulation in time domain.

PACS numbers: $71.55 . \mathrm{Eq}, 72.20 . \mathrm{Jv}$

\section{Introduction}

In highly-excited semiconductors, dynamics of carriers in temporally and spatially varying space-charge (SC) electric field may lead to nonlinear effects which can be detected by various optical techniques. The previous studies of carrier transport and recombination by a picosecond four-wave mixing (FWM) technique in semi-insulating vanadium doped CdTe crystals revealed oscillationdecorated decay of the diffraction efficiency of electrooptic and free carrier (FC) gratings [1]. The origin of these oscillations was attributed to acoustic waves, which were generated by spatially-modulated electric field via an elastooptic effect and propagated along the grating vector with sound velocity.

Here we extended the nonlinear optical studies of nonequilibrium processes in nonuniformly excited semi-insulating InP:Fe crystal. The measurements of timeresolved FWM on FC grating, combined with numerical calculations, have shown a novel origin of the oscillations in subnanosecond time-domain. It was attributed to effect of simultaneous presence of hole and electron gratings, which drift in the SC field and contribute to refractive index modulation accordingly to their relative phase, which changes with time.

\section{Experimental technique and model}

The optical nonlinearities were investigated in highly-excited InP bulk crystal using time-resolved degenerate FWM technique [2]. The measured wafer was cut from (100) boule, grown by the liquid encapsulated Czochralski method. For 
compensation, the wafer was slightly doped with iron impurity, which forms a relevant deep $\mathrm{Fe}^{2+} / \mathrm{Fe}^{3+}$ levels [3]. Nonequilibrium charge carriers were generated by an interference pattern of two $25 \mathrm{ps}$ duration laser pulses at $1064 \mathrm{~nm}$ wavelength, thus involving Fe-impurity related transitions and forming the FC and $\mathrm{SC}$ gratings. In these conditions, the FC nonlinearity is dominant mechanism of refractive index modulation, even at rather small spacings of the interference pattern. The contribution of SC electric field is usually revealed in carrier drift, which in monopolar case opposes the diffusive decay of a transient FC grating with a small spatial period. The subnanosecond dynamics of FC grating was monitored by varying the probe-beam delay $\Delta t$ from 0 to $2 \mathrm{~ns}$.

Theoretical analysis of the entire process of transient grating formation and decay was based on a conventional set of the continuity and rate equations, written for semi-insulating InP:Fe model that accounts for the generation and recombination processes via deep $\mathrm{Fe}^{2+} / \mathrm{Fe}^{3+}$ levels and excited $\mathrm{Fe}^{2+*}$ state. For numerical simulations, the following InP:Fe crystal parameters at wavelength $\lambda=1064 \mathrm{~nm}$ were used [3]: the initial density of ionized and neutral iron impurities $\left[\mathrm{Fe}^{2+}\right]=1 \times 10^{15} \mathrm{~cm}^{-3}$ and $\left[\mathrm{Fe}^{3+}\right]=1.5 \times 10^{16} \mathrm{~cm}^{-3}$, the photoionization cross-sections for the electrons $s_{\mathrm{e}}=5 \times 10^{-17} \mathrm{~cm}^{2}, s_{\mathrm{e}}^{*}=5.35 \times 10^{-17} \mathrm{~cm}^{2}$ and holes $s_{\mathrm{p}}=1.7 \times 10^{-17} \mathrm{~cm}^{2}, s_{\mathrm{p}}^{*}=3.3 \times 10^{-17} \mathrm{~cm}^{2}$, and the capture coefficients for the electrons $\gamma_{\mathrm{e}}=4.1 \times 10^{-8} \mathrm{~cm}^{3} / \mathrm{s}, \gamma_{\mathrm{e}}^{*}=2 \times 10^{-7} \mathrm{~cm}^{3} / \mathrm{s}$, and holes $\gamma_{\mathrm{p}}=\gamma_{\mathrm{p}}^{*}=1.6 \times 10^{-8} \mathrm{~cm}^{3} / \mathrm{s}$.

We analyzed the case when spatially modulated light pattern creates the index grating through the FC nonlinearity. The calculated spatial and temporal variation of carrier densities were used to extract the relevant time dependence of the index modulation by the electron grating $\Delta n_{\mathrm{e}}(t) \sim\left(N / m^{*}\right)_{1}$, by the hole grating $\Delta n_{\mathrm{p}}(t) \sim\left(P / m_{\mathrm{p}}\right)_{1}$, and by the FC grating $\Delta n_{\mathrm{fc}}(t)=C_{\mathrm{fc}}\left(N / m^{*}+P / m_{\mathrm{p}}\right)_{1}$, here $N, m^{*}$ and $P, m_{\mathrm{p}}$ are the density and the effective mass of electrons and holes, $C_{\mathrm{fc}}$ is the modulation coefficient, and the subscript "1" means the fundamental spatial harmonics which governs the Bragg diffraction. The dynamics of optical nonlinearities was analyzed using the simulated instantaneous carrier and field distributions of optically modulated structure.

\section{Results and discussion}

Figure 1 shows the probe-beam diffraction efficiency simulated at different external conditions of time-resolved FWM. The plots demonstrate that the oscillatory behavior of FC grating decay appears at higher excitations (Fig. 1a) and is more pronounced for smaller grating periods (Fig. 1b), after the diffusion erases the FC density modulation and builds up the SC field. Moreover, the oscillations reshape a dependence of the diffraction efficiency on excitation intensity (Fig. 1c), if the FWM response is measured at later times of the probe-beam delay $\Delta t \gg t_{1}$ (here $t_{1}$ is the laser pulse duration).

The origin of oscillations is clearly different from the previously observed ones [1] and can be attributed to peculiarities of different type carrier transport in 


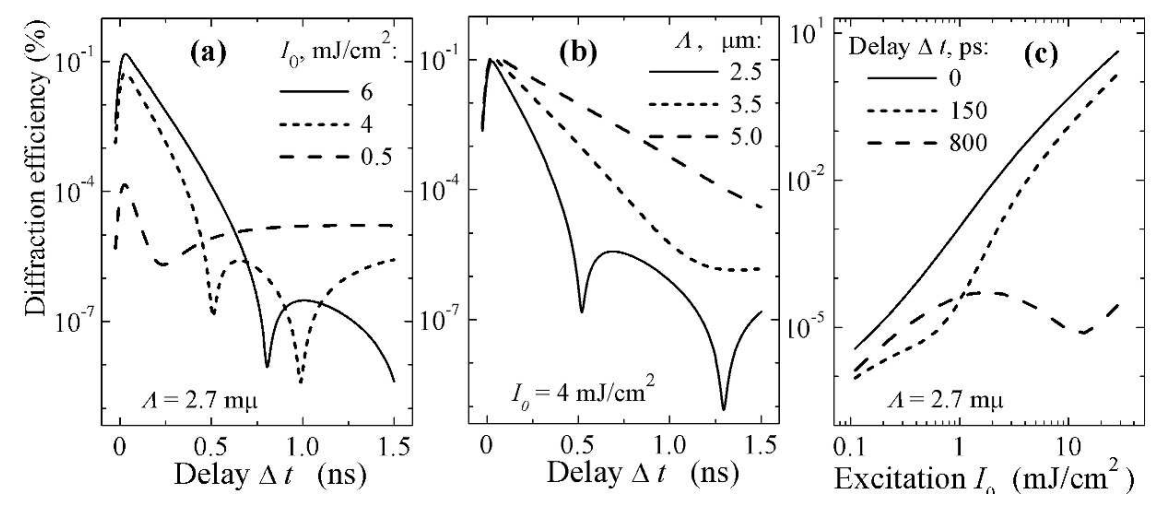

Fig. 1. Diffraction efficiency vs. (a),(b) probe-beam delay $\Delta t$ and (c) excitation intensity $I_{0}$, simulated for the period $\Lambda$ using modulation coefficient $C_{\mathrm{fc}}=-3.5 \times 10^{-22} \mathrm{~cm}^{3}$.
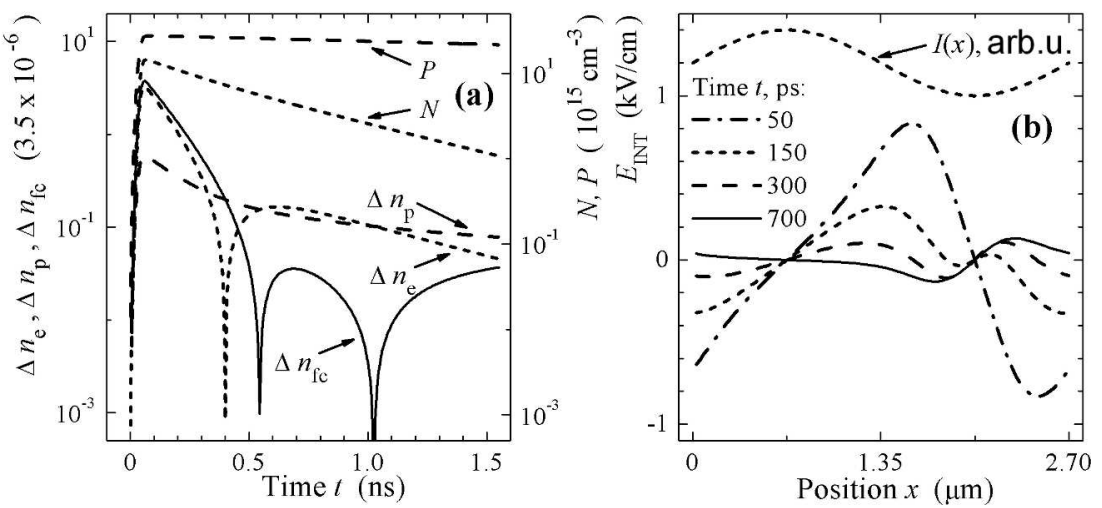

Fig. 2. (a) Time dependence of refractive index modulation by free carriers $\Delta n_{\mathrm{fc}}$, electron grating $\Delta n_{\mathrm{e}}$ and hole grating $\Delta n_{\mathrm{fc}}$, and dynamics of carriers density ( $N$ and $P$, right-side scale), simulated for the period $\Lambda=2.7 \mu \mathrm{m}$ and excitation $I_{0}=4 \mathrm{~mJ} / \mathrm{cm}^{2}$. (b) Spatial distribution of internal electric field at time $t$; here $I(x)=I_{0} \sin (2 \pi x / \Lambda)$ provides the shape of the interference pattern.

SC electric field and their varying with time contribution to refractive index modulation. For better understanding, in Fig. 2a we compare the evolution of refractive index modulation for each carrier grating. In addition, Fig. $2 \mathrm{~b}$ demonstrates the spatial distribution of internal electric field $E_{\text {int }}$ at different time moments $t$. Oscillatory behavior in the $\Delta n_{\mathrm{fc}}$ kinetics appears when the contribution of electrons and holes to the index modulation become comparable and the phase shift of the electron grating, with respect to the hole grating, changes from 0 to $\pi$. Experimentally, time-resolved FWM was carried out on the FC grating with $\Lambda=2.7 \mu \mathrm{m}$ by varying the probe-beam delay and excitation intensity (Fig. 3). The oscillations were observed at higher excitation levels and appear at slightly longer probe-beam delay, as it was predicted numerically. The oscillations were not present at low 

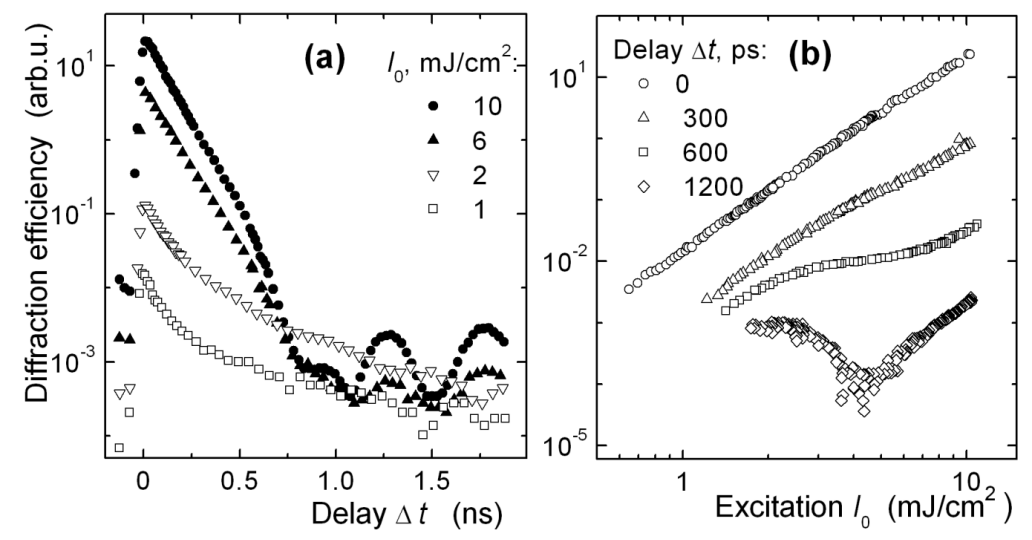

Fig. 3. Experimental results of probe-beam diffraction efficiency vs. (a) the probe beam delay $\Delta t$ and (b) excitation energy density $I_{0}$, measured for the grating with $\Lambda=2.7 \mu \mathrm{m}$.

excitations because of a rather weak SC electric field in the given crystal with not known initial occupation of the impurity levels. Nevertheless, at higher excitations $\left(6-10 \mathrm{~mJ} / \mathrm{cm}^{2}\right)$ and later time $(\Delta t>1 \mathrm{~ns})$, the SC field was high enough for electron grating to drift and contribute to diffraction jointly with the hole grating, adding constructively or destructively. The oscillating kinetics reshaped the diffraction efficiency as well at $\Delta t>0.6 \mathrm{~ns}$ (Fig. 3b), in agreement with the simulation results (Fig. 1c).

\section{Conclusions}

The study performed has proved a novel origin of the oscillations in subnanosecond carrier dynamics, seen in highly excited InP:Fe crystals by timeresolved FWM technique. The numerical analysis of electron and hole density modulation, its localization and transport in varying with time space charge electric field provided the deeper understanding of nonequilibrium carrier dynamics in highly-excited and spatially-modulated structure. The technique can be applied for evaluation of deep trap photoexcitation and formation of the fast space charge oscillations in compensated semiconductors.

\section{References}

[1] D. Verstraeten, M.L. Hellin, Ph.L. Lemaire, A. Kadys, M. Sudzius, K. Jarasiunas, in: Photorefractive Effects, Materials, and Devices, Eds. G. Zhang, D. Kip, D. Nolte, J.J. Xu, OSA TOPS 99, 255 (2005).

[2] A. Kadys, M. Sudžius, K. Jarašiūnas, L. Mao, N. Sun, Mater. Sci. Eng. B 133, $136(2006)$.

[3] A. Kadys, L. Subačius, R. Aleksiejūnas, K. Jarašiūnas, Proc. SPIE 6595, 659615 (2007). 